\title{
Estudio detallado de las causas de transferencia de Diálisis Peritoneal a hemodiálisis en un Servicio de Nefrologia
}

\author{
C. Blasco Cabañas \\ E. Ponz Clemente \\ N. Mañe Buixo \\ JC. Martínez Ocaña \\ D. Marquina Parra \\ E. Yuste Giménez \\ M. García García
}

Servicio de Nefrología
Corporación Parc Taulí
Sabadell (Barcelona)

\section{RESUMEN}

Objetivo: Analizar las causas y los factores de riesgo de transferencia a HD de un programa de DP.

Material y métodos: Estudio retrospectivo y descriptivo. La DP se ofrece en un plano de igualdad a todos los enfermos con IRCT.

Se han recogido variables demográficas, clínicas, existencia de deficiencias físicas, autonomía funcional, peritonitis, tiempo de seguimiento y motivo de finalización de la técnica; causa de transferencia inmediata a hemodiálisis, clasificándolas en potencialmente solucionables o no.

De los enfermos transferidos se analizaron: datos clínicos, estado funcional y datos sociales.

Resultados: De un total de 103 pacientes, un $58,25 \%$ varones, con una edad media al inicio de la DP de $59 \pm 18$, se han transferido a HD un
$29 \%$, se han trasplantado un $16,5 \%$ y fallecieron el 29\%, mientras que 26 enfermos siguen en programa.

Las causas inmediatas del paso a HD fueron: fallo de ultrafiltración (UF) y/o infradiálisis 30\%, peritonitis $17 \%$, problemas relacionados con el catéter o la pared abdominal $17 \%$, intolerancia a la DP $17 \%$, problema social $y / 0$ enfermedad grave $13 \%$ y deseo del paciente $6 \%$.

Del total de transferencias, el 46,7\% eran por problemas relacionados con la DP no solucionables.

Los pacientes transferidos tuvieron más peritonitis que el resto y una mayor proporción de deficiencias físicas que dificultaban realizar la DP. Se observó una gran influencia de los factores personales y sociales en la supervivencia de la técnica de DP.

\section{Correspondencia:}

Conchita Blasco

Servicio de Nefrología

Corporación Parc Taulí s/n

08207 Sabadell. Barcelona
PALABRAS CLAVE: DIÁLISIS PERITONEAL HEMODIÁLISIS CAMBIO DE TRATAMIENTO 


\section{A DETAILED STUDY OF THE CAUSES OF TRANFERENCE FROM PERITONAL DIALYSIS TO HEMODIALYSIS IN A NEPHROLOGY SER- VICE.}

\section{SUMMARY}

Objective: To analyse the causes and risk factors of transference to HD in a programme of PD.

Material and methods: A descriptive and retrospective study. PD is offered across the board to all patients with IRCT.

The following variable were gathered: demographics, clinics, existence of physical deficiencies, functional autonomy, peritonitis, time of treatment and reason for stopping the technique; immediate causes of transfer to hemodialysis, classified as potentially solvable or not.

We analyzed the following data on the patients: clinical data, funcional status and social data.

Results: Of a total of 103 patients $58.25 \%$ of them men, with an average age at the beginning of PD of $59 \pm 18,29 \%$ transfered to HD, $16.5 \%$ had transplants, $29 \%$ died, while 26 people continue in the programme.

The immediate causes of transfer to HD were: $30 \%$ failure of ultrafiltration (UF) and / or infradialysis, $17 \%$ peritonitis, $17 \%$ problems related to the catheter or the abdominal wall, $17 \%$ intolerance of PD, $13 \%$ social problem and / or serious illness and $6 \%$ the wish of the patient.

Of the total transfers, $46 \%$ were due to problems related to $\mathrm{PD}$ for which there was no solution.

The transfer patients had more peritonitis than others and a major proportion of physical deficiencies which made it difficult to carry out PD. Personal and social factors were observed to have a strong influence on the survival of the technique of PD.

\section{KEY WORDS:}

\section{PERITONEAL DIALYSIS}

HEMODIALYSIS

CHANGE OF TREATMENT
En la mayoría de revisiones bibliográficas se considera que la peritonitis (29-54\% de los casos) es la primera causa de transferencia de diálisis peritoneal (DP) a hemodiálisis (HD), seguida del fallo de ultrafiltración y del transporte peritoneal (9-29\% de los casos). A menudo, el fallo de la técnica de DP se atribuye a infradiálisis, sin llegar a optimizar la prescripción de DP.

Los registros generales de resultados de las técnicas de diálisis recogen con frecuencia las causas de cambio de técnica, pero a veces es difícil clasificar en un apartado el motivo de la transferencia. De ahí la utilidad de estudios detallados en un único centro, donde puede catalogarse mejor la causa de transferencia y además clasificarla como potencialmente solucionable o no.

Existe también una gran preocupación sobre la supervivencia de la técnica que es claramente inferior a la de $\mathrm{HD}$, aunque no conocemos con exactitud cuando se ha agotado la membrana peritoneal y motivos médicos irresolubles impiden la continuación de la DP. Sin embargo, hay pacientes con más de 5 años en DP y sin función renal residual que mantienen intacta su capacidad dialítica ${ }^{(1)}$. Se han descrito grupos de enfermos con largas permanencias en DP y el análisis de las características de estos enfermos concluye que se trata de enfermos de tamaño medio, muy cumplidores, con una baja tasa de peritonitis, entusiastas de la DP, transportadores medios $y$ con un alto soporte social(2).

Los resultados de la DP dependen de la experiencia del centro, del entusiasmo y esfuerzo del equipo de $\mathrm{DP}^{(3)}$. Cuanto mayor es el número de enfermos tratados menor es la tasa de transferencia(4).

El desarrollo de protocolos asistenciales es de gran ayuda para los programas domiciliarios como es la $\mathrm{DP}^{(5)}$. El mantener un nivel de auto cuidados adecuado en el entorno habitual del paciente requiere un desarrollo del programa de entrenamiento y educación ${ }^{(6)}$.

Recientemente algún estudio de supervivencia de la técnica de DP ya sugiere que otros factores, como complicaciones derivadas de la comorbilidad de estos enfermos y la problemática social, representan las causas principales de cambio de técnica ${ }^{(4)}$.

\section{OBJETIVO}

Analizar las causas y los factores de riesgo de transferencia de los pacientes en un programa de DP a HD.

\section{MATERIAL Y MÉTODOS}

Hemos realizado un estudio retrospectivo y descriptivo de la evolución del programa de Diálisis Peritoneal desde su creación en septiembre de 1991 hasta el 31 de oc- 
tubre de 2002. El programa de DP se inició junto con el programa de atención a la Insuficiencia Renal Crónica Terminal (IRCT) en un Hospital general que no disponía hasta entonces de atención Nefrológica. Desde la creación del Servicio de Nefrología, la DP se ofreció en plano de igualdad a todos los enfermos con IRCT, salvo que tuvieran contraindicaciones para alguna de las técnicas, a través de un programa informativo estructurado. El objetivo de este programa informativo sobre las técnicas de diálisis ha sido siempre ayudar a la elección de la técnica de diálisis más adecuada para cada enfermo.

Se han recogido al inicio de la DP variables demográficas (edad, género), clínicas (talla, peso, nefropatia, función renal residual, modalidad de DP, diabetes), existencia de deficiencias físicas (visuales, de habilidad manual o de movilidad), autonomía funcional (Karnofsky modificado), persona que realizaba la técnica (enfermo, cónyuge, hijos u otras personas) y el porcentaje de pacientes que eran sus propios cuidadores. Se han recogido también: peritonitis (tasa de peritonitis y meses hasta la primera peritonitis), tiempo de seguimiento y motivo de finalización de la técnica, causa de muerte, causa de transferencia a hemodiálisis.

Las causas de transferencia a HD se clasificaron en potencialmente solucionables o no. Se consideraban solucionables cuando después de un motivo de transferencia a HD el enfermo no reiniciaba DP sin motivo médico que contraindicara la técnica, es decir, motivos sociales y personales hacían que el enfermo no volviera a DP. De los enfermos transferidos a hemodiálisis se analizaron en el momento de la transferencia: peso, prescripción de diálisis, adecuación de diálisis (KT/Vs y $\mathrm{ClCr} / 1.73 \mathrm{~m}^{2}$ ), estado funcional y datos sociales (soporte familiar). También se recogió la valoración del estado del domicilio y de la realización de la técnica mediante unos cuestionarios específicos utilizados en la unidad, la escala de puntuación del domicilio oscila entre 0-18 puntos y la realización de la técnica entre 0-30. Para el estudio estadístico se ha utilizado el paquete estadístico SPSS. Se han expresado las variables numéricas como media y desviación típica. Se han aplicado las pruebas de $\mathrm{Chi}^{2} \mathrm{y} \mathrm{T}$ de Student. El análisis de la supervivencia de la técnica, del enfermo y del tiempo libre de peritonitis se ha realizado mediante curvas de supervivencia actuarial de log-rank. La significancia se ha definido como una $\mathrm{p}<0,05$.

\section{RESULTADOS}

Se han tratado un total de 103 pacientes, un 58,25\% eran varones, con una edad media al inicio de la DP de $59 \pm 18$ años. Las características generales de los enfermos quedan reflejadas en la tabla 1.

\begin{tabular}{|l|l|}
\hline \multicolumn{2}{|c|}{ Características de los pacientes en DP } \\
\hline № Total de pacientes & 103 \\
\hline Hombres/Mujeres (n) & $60 / 43$ \\
\hline Diabéticos (\%) & $32 \%$ \\
\hline Edad inicio DP (años, Media \pm DS [rango]) & $59.0 \pm 17.8(14.2-89.2)$ \\
\hline Peso inicio DP (Kg, Media \pm DS [rango]) & $69.2 \pm 11.4(36-101)$ \\
\hline IMC inicio DP (kg/m², Media \pm DS [rango]) & $25.6 \pm 4.0(15.8-34.9)$ \\
\hline Días en DP hasta 1ª peritonitis (Mediana) & 512 \\
\hline Tasa peritonitis (meses-paciente por peritonitis) & 19.4 \\
\hline DPCA/DPA (n) & $51 / 52$ \\
\hline Meses en DP (Media \pm DS) & $21.6 \pm 18.0$ \\
\hline Pacientes más de 3 años en DP & $20.4 \%$ \\
\hline
\end{tabular}

Tabla 1: Características de los pacientes en DP.

Cuatro enfermos $(3,88 \%)$ habían sido transferidos de $\mathrm{HD}$, dos por problemas de acceso vascular y dos por deseo del enfermo; siete enfermos (6,79\%) procedían de trasplante renal y todos ellos optaron por la DP sin contraindicaciones para la HD.

Durante estos 11 años se han transferido a HD 30 pacientes (29\%), que llevaban en DP 19,8 \pm 15 meses, se han trasplantado 17 pacientes (16,5\%), 30 enfermos (29\%) fallecieron tras permanecer en DP $18,8 \pm 15$ meses y 26 enfermos siguen en programa.

Las causas inmediatas del cambio a HD fueron: fallo de ultrafiltración (UF) y/o infradiálisis en 9 enfermos (30\%), peritonitis en 5 enfermos (17\%), problemas relacionados con el catéter o la pared abdominal en 5 enfermos (17\%), intolerancia a la DP en 5 casos (17\%), problema social y/o enfermedad grave en 4 casos (13\%) y deseo del paciente sin otro motivo en 2 enfermos (6\%). En ningún caso se retiró un catéter por infección del orificio o del túnel de manera aislada. En los 5 casos de problemas relacionados con el catéter los problemas fueron: una fuga pleural, una fuga genital, un segundo reatrapamiento del catéter por el epiplón, una caída espontánea del catéter por despegamiento de los topes de dacron en un catéter de poliuretano y una hernia incarcerada con retirada del catéter durante la cirugía.

Del total de transferencias, 14 casos $(46,7 \%)$ eran por problemas relacionados con la DP no solucionables: 5 por intolerancia, 3 por fallo de la UF, 3 por infradiálisis, 1 fuga pleural, 1 reatrapamiento por epiplón y solo un caso de peritonitis con perforación intestinal y acceso crónico intrabdominal. Se consideró infradiálisis no solucionable cuando el enfermo realizaba DP automática con cicladora como mínimo día húmedo y un intercam- 
bio diurno. En los 16 casos restantes, motivos médicos, sociales o personales influyeron en el cambio definitivo de técnica, sin existir contraindicaciones absolutas para continuar o reintroducir la DP. Figura 1.

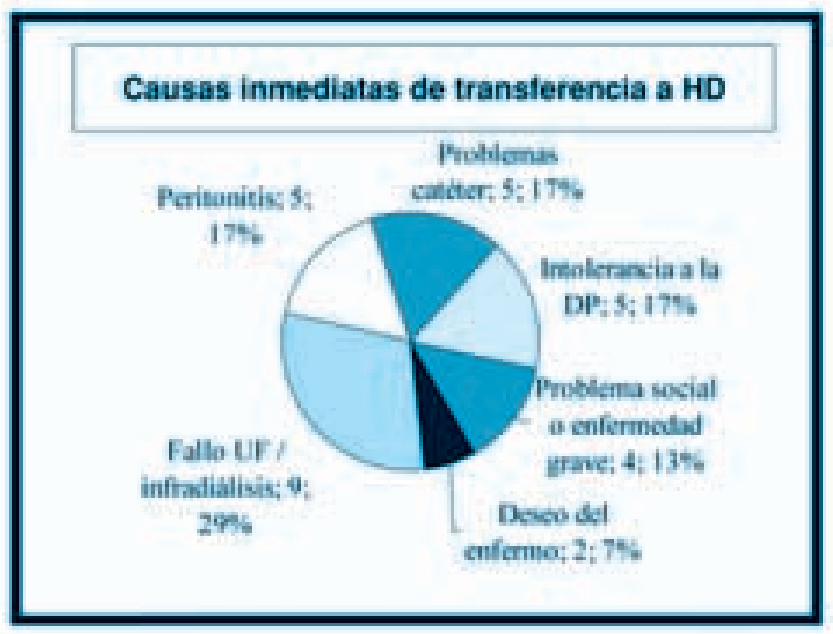

Figura 1: Causas inmediatas de transferencia a HD.

De los pacientes trasferidos, 17 (56,70\%) pasaron a HD antes de haber trascurrido un año tras su inclusión en DP. En 5 casos el motivo fue la intolerancia a la téc- nica, con molestias abdominales, anorexia y mal estado general no relacionado con una dosis de diálisis inadecuada. En dos casos, los enfermos decidieron por voluntad propia pasar a $\mathrm{HD}$, al mes y dos meses de su inclusión, al reconocer que habían equivocado la elección. Seis pacientes (20\%) fueron transferidos tras más de 3 años en DP.

$\mathrm{Si}$ analizamos el origen de estos pacientes observamos que para 24 pacientes (80\%) la DP era su primera técnica de diálisis; 4 pacientes $(13,5 \%)$ provenían de transplante renal y solo 2 pacientes $(6,5 \%)$ de hemodiálisis. En la tabla 2 se resumen las características de los pacientes transferidos a HD comparados con los no transferidos. Los pacientes transferidos a HD tuvieron más peritonitis que el resto ya que la tasa de peritonitis fue de 14.5 vs 22,0 meses-paciente/peritonitis $(P=0,002)$, y hubo una mayor proporción de deficiencias físicas que dificultaban realizar la DP $60 \%$ vs $47 \%(\mathrm{P}=0,007)$.

En la figura 2 se muestran las curvas de supervivencia libres de peritonitis de los dos grupos de enfermos. La mediana de días para los transferidos fue de 422 y para los no transferidos de 545 días, aunque la diferencia no fue signicativa.

No se observaron diferencias estadísticamente significativas entre los pacientes transferidos a HD y el resto en cuanto a la edad, género, meses en DP, índice de masa corporal y nefropatía de base.

\begin{tabular}{|c|c|c|c|}
\hline \multicolumn{4}{|c|}{ Características de los pacientes en DP transferidos a HD Vs los no transferidos } \\
\hline & $\begin{array}{c}\text { Transferidos a HD } \\
\quad(\mathrm{n}=30)\end{array}$ & $\begin{array}{c}\text { No transferidos } \\
\qquad(\mathrm{n}=73)\end{array}$ & $\mathrm{P}(*)$ \\
\hline Edad al iniciar DP & $59,6 \pm 15,7(22-80)$ & $58,6 \pm 18,3(14-89)$ & NS \\
\hline$\%$ mayores de 70 años & $40 \%$ & $34,2 \%$ & NS \\
\hline Sexo (\% hombres) & $50 \%$ & $61,6 \%$ & NS \\
\hline $\mathrm{IMC}$ inicio $\mathrm{DP}(\mathrm{Kg} / \mathrm{m} 2, \mathrm{X} \pm \mathrm{DS})$ & $26,9 \pm 5,3$ & $25,8 \pm 5,0$ & NS \\
\hline $\begin{array}{l}\text { Técnica: } \\
\text { \% DPCA } \\
\% \text { DPA }\end{array}$ & $\begin{array}{l}46,7 \% \\
53,3 \%\end{array}$ & $\begin{array}{l}50,6 \% \\
49,4 \%\end{array}$ & NS \\
\hline Meses en DP $(\mathrm{X} \pm \mathrm{DS})$ & $19,8 \pm 21,8$ & $22,3 \pm 16,3$ & NS \\
\hline $\begin{array}{l}\text { Tasa peritonitis } \\
\text { (meses-paciente por peritonitis) }\end{array}$ & 14,5 & 22,0 & 0,002 \\
\hline$\%$ con deficiencias físicas (manuales, visuales, otras) & $60 \%$ & $47 \%$ & 0,007 \\
\hline $\begin{array}{l}\text { Autonomía funcional: } \\
\% \text { pacientes dependientes o que requerían atención } \\
\text { continuada u hospitalización }\end{array}$ & $30 \%$ & $26 \%$ & NS \\
\hline $\begin{array}{l}\text { Quién realiza la DP } \\
\text { - Paciente } \\
\text { - Cónyuge } \\
\text { - Hijos / otros }\end{array}$ & $\begin{array}{c}70 \% \\
23,3 \% \\
6,7 \%\end{array}$ & $\begin{array}{l}60,3 \% \\
24,7 \% \\
15,1 \%\end{array}$ & NS \\
\hline \% en que el propio paciente es el cuidador de sí mismo & $40 \%$ & $35,6 \%$ & NS \\
\hline
\end{tabular}

Tabla 2: Características de los pacientes. 


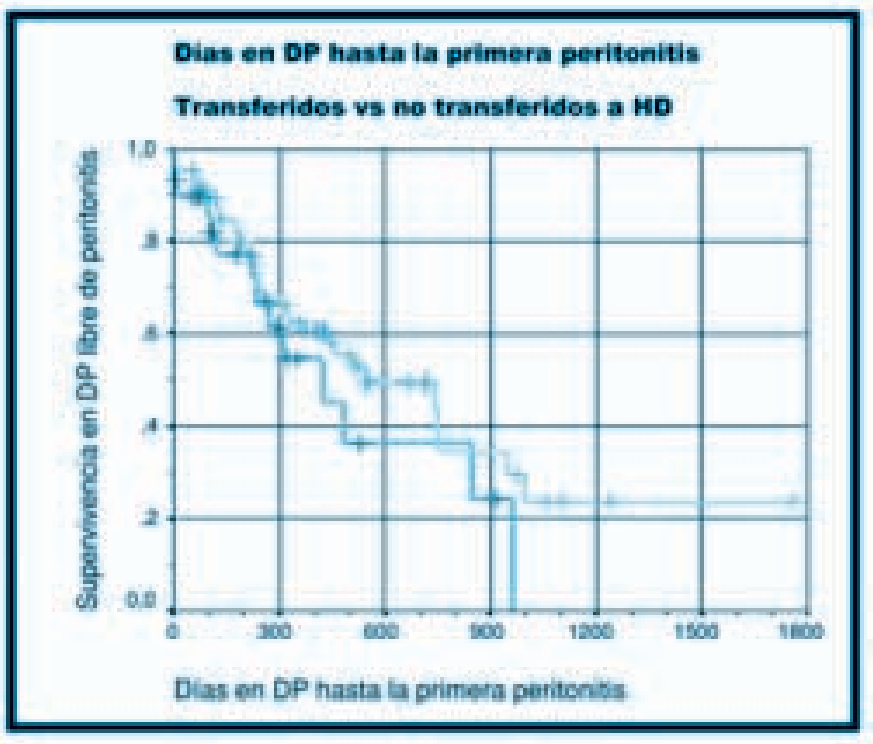

Figura 2: Días en DP hasta la primera peritonitis.

Una peor autonomía funcional, una mayor dependencia física y la falta de soporte familiar se asociaban a la transferencia a HD, aunque las diferencias no eran estadísticamente significativas.

En cuanto a la valoración del domicilio destacar que en 19 pacientes obtuvieron la puntuación máxima (18 puntos), 10 pacientes oscilaron entre 13 y 17 puntos y solo en 1 caso la puntuación era de 5 puntos, es decir que precisaba mejorar las condiciones del domicilio.

La valoración de la técnica en 19 pacientes es de 30 puntos y en 11 casos se encuentran entre 24-29 puntos.

La media de supervivencia de la técnica fue de 49.1 mes y la mediana de 65.3 meses. Entre los pacientes fallecidos o transferidos a HD en el primer año tras el inicio de la DP había un mayor porcentaje de diabéticos (45\% vs $24 \%)$.

\section{DISCUSIÓN}

La causa más frecuente de fallo de la técnica de DP en nuestra experiencia no ha sido la peritonitis ni problemas infecciosos relacionados con el catéter. En 5 casos la peritonitis fue la causa inmediata de transferencia a HD y solo en un caso complicado con una perforación intestinal y un acceso pélvico no pudo plantearse el retorno a DP. En los otros 4 casos, motivos personales, deterioro del estado general y falta de soporte social motivaron que se mantuvieran en HD.

La mayoría de estudios de causas de transferencia donde la peritonitis es la primera causa son de finales de los años 80 y principios de los 90 . Actualmente, con la me- joría de los materiales para DP y de las conexiones, así como de la experiencia acumulada en los programas de $\mathrm{DP}$, las tasas de peritonitis han disminuido notablemente, por lo que parece lógico que ésta no sea ya la primera causa. Nuestro programa empezó en 1991 y pocos años después ya se generalizó el uso de los sistemas de doble bolsa, y desde el año 1996 el uso de la cicladora se ha incrementado notablemente.

Aunque la principal causa individual de cambio de técnica ha sido la infradiálisis y/o el fallo de ultrafiltración (30 \%), en realidad, solo había 3 casos de verdadero fallo de membrana con pérdida de UF. Dos de estos enfermos realizaron un reposo peritoneal de 2 meses sin mejoría y se transfirieron definitivamente a $\mathrm{HD}$, uno recibió un TR y está en buen estado de salud 4 años después, y la otra enferma lleva un año en HD. El tercer paciente que pasó a HD por fallo de UF y en el contexto de enfermedad grave con amputaciones y malnutrición, se diagnosticó al año de pasar a HD de una peritonitis esclerosante y falleció a los pocos meses. De los 6 pacientes que sufrían infradiálisis, en tres de ellos no se había apurado la prescripción de diálisis, es decir, no habían pasado a DP automática desde DPCA, o no habían realizado un intercambio diurno.

Con relación a las causas de fallo temprano de la técnica, la más frecuente es la intolerancia a la DP que a veces puede enmascarar un fallo de elección de la técnica, aunque en algún caso parecía existir una auténtica voluntad por permanecer en DP, tanto por parte del enfermo como de su familia.

De los 16 casos que no se apuraron las posibilidades terapéuticas de la DP, los factores que más influyeron en el cambio definitivo de la técnica fueron la falta de soporte familiar en enfermos que se habían deteriorado y ya no eran autónomos. Según refiere la bibliografía, la incidencia de la necesidad de un familiar de apoyo en la técnica de diálisis peritoneal es alta, cercana a la quinta parte de los pacientes ${ }^{(7)}$.

La valoración del domicilio y de la técnica no es un dato significativo con relación a la transferencia, pero si muy importante para poner de relevancia posibles carencias que precisen de una actuación por parte del equipo asistencial, cabe destacar que el paciente que no supere una puntuación mínima no puede iniciar la técnica en el domicilio.

Si analizamos dos periodos de tiempo, de 1992 a 1996 y de 1997 a 2002, el número de enfermos transferidos ha sido similar. En el primer periodo, 4 enfermos pasaron a HD por intolerancia o propia elección, mientras que en el segundo periodo, solo 2 enfermos fueron transferidos por ese motivo. Es posible que la experiencia del personal en la información sobre las técnicas de diálisis y la mejoría de las técnicas de comunicación con el enfermo y sus familiares disminuyan los errores en la elección. 
El análisis de estos datos refleja que nuestro programa ha adquirido experiencia; no solemos tener problemas de colocación del catéter, no hay fugas, no se cae el catéter. Y los problemas actuales suelen ser los que encontramos en la bibliografía, pacientes con problemas sociales, con deterioro físico y multipatologia, que van a dificultar su permanencia en diálisis peritoneal.

La supervivencia de la técnica depende también, en gran medida, del entusiasmo del equipo médico y de enfermería responsable del programa de DP, que pueden pensar, ofertar posibilidades y pautas individualizadas y terapias flexibles.

Por ultimo, creemos que es importante saber dónde esta el límite de la técnica, ya que si se mantiene la técnica en situación de infradiálisis o dificultad para mantener un correcto peso seco, puede ocasionar un perjuicio para el enfermo y aumentar la morbi-mortalidad. Al reflexionar sobre los límites de la técnica no podemos olvidar la presión emocional y psicológica que puede ocasionar la DP a las familias, cuidadores y pacientes.

\section{CONCLUSIONES}

1) La peritonitis no fue la primera causa de transferencia a HD, aunque los pacientes transferidos tenían más peritonitis.

2) Si bien un $30 \%$ se transfirieron por infradiàlisis o fallo de la UF, sólo en un 10\% era debido a un fallo real de la membrana.

3) Se observó una gran influencia de los factores personales y sociales en la supervivencia de la técnica de DP.

4) Los 5 pacientes que intoleraron la DP se transfirieron a HD entre 0,1 y 8 meses tras la inclusión, lo que sugeriría un problema de elección de la técnica.

\section{BIBLIOGRAFÍA}

1. Hevia C, Bajo MA, Peso G, Sánchez-Tomero JA, Fernández MJ, Selgas R. Efecto de las características funcionales peritoneales básales en la supervivencia del paciente y de la técnica. El estado de alto transportador no influye en ninguna de ellas. Libro de Comunicaciones de la IV Reunión Nacional de Diálisis Peritoneal. Barcelona, enero 2003. Pág. 26.

2. Díaz-Buxo JA, Shultman DS. Characteristics of longterm peritoneal dialysis patients. Adv Pert Dial 1997; 13: $104-8$.

3. Schaubel DE, Blake PG, Fenton SS. Effect of renal center characteristics on mortality and technique failure on peritoneal dialysis. Kidney Int 2001 Oct; 60(4): 1517-24.

4. Rodríguez-Carmona A, García Falcón T, Pérez Fon$\tan$ M, Bouza P, Adeva M, Rivera CF, Valdes F. Survival on chronic peritoneal dialysis: have results improved in the 1990s?. Perit Dial Int 1996; 16 Suppl 1: S410- 3.

5. Luque E, Barroso R, López M, Alapont M, Alconchel S. Diálisis peritoneal, la realidad subjetiva de convivir con una enfermedad crónica. Revista de la Sociedad Española de Enfermaría Nefrológica 2001; 13:1217.

6. Gómez A C, Sánchez J, Ojeda A. Programa de entrenamiento de diálisis peritoneal. La visita domiciliaria. Experiencia de nueve años. Revista de la Sociedad Española de Enfermería Nefrológica 2000; 9: 6-10.

7. Serrato F, Pérez A, Oua M, Ramos J. Diálisis peritoneal: cuando la técnica depende del familiar. Libro de Comunicaciones del XXV Congreso Nacional de la SEDEN. Oviedo 2000. Pág. 96. 